\title{
Role of insulin in the regulation of human skeletal muscle protein synthesis and breakdown: a systematic review and meta-analysis
}

\author{
Haitham Abdulla $^{1} \cdot$ Kenneth Smith $^{1} \cdot$ Philip J. Atherton $^{1} \cdot$ Iskandar Idris $^{1}$
}

Received: 24 April 2015 / Accepted: 19 August 2015 / Published online: 24 September 2015

(C) Springer-Verlag Berlin Heidelberg 2015

\begin{abstract}
Aims/hypothesis We aimed to investigate the role of insulin in regulating human skeletal muscle metabolism in health and diabetes.

Methods We conducted a systematic review and metaanalysis of published data that examined changes in skeletal muscle protein synthesis (MPS) and/or muscle protein breakdown (MPB) in response to insulin infusion. Random-effects models were used to calculate weighted mean differences (WMDs), 95\% CIs and corresponding $p$ values. Both MPS and MPB are reported in units of nmol $(100 \mathrm{ml} \mathrm{leg} \mathrm{vol.})^{-1}$ $\min ^{-1}$.

Results A total of 104 articles were examined in detail. Of these, 44 and 25 studies (including a total of 173 individuals) were included in the systematic review and meta-analysis, respectively. In the overall estimate, insulin did not affect MPS (WMD 3.90 [95\% CI $-0.74,8.55], p=0.71$ ), but significantly reduced MPB (WMD -15.46 [95\% CI -19.74 , $-11.18], p<0.001)$. Overall, insulin significantly increased net balance protein acquisition (WMD 20.09 [95\% CI 15.93, 24.26], $p<0.001$ ). Subgroup analysis of the effect of insulin on MPS according to amino acid (AA) delivery was performed using meta-regression analysis. The estimate size (WMD) was significantly different between subgroups based
\end{abstract}

Electronic supplementary material The online version of this article (doi:10.1007/s00125-015-3751-0) contains peer-reviewed but unedited supplementary material, which is available to authorised users.

Iskandar Idris

Iskandar.Idris@nottingham.ac.uk

1 Division of Medical Sciences \& Graduate Entry Medicine, School of Medicine, University of Nottingham, Royal Derby Hospital,

Uttoxeter Road, Derby DE22 3DT, UK on AA availability $(p=0.001)$. An increase in MPS was observed when AA availability increased (WMD 13.44 [95\% CI 4.07, 22.81], $p<0.01$ ), but not when AA availability was reduced or unchanged. In individuals with diabetes and in the presence of maintained delivery of AA, there was a significant reduction in MPS in response to insulin (WMD -6.67 [95\% CI $-12.29,-0.66], p<0.05$ ).

Conclusions/interpretation This study demonstrates the complex role of insulin in regulating skeletal muscle metabolism. Insulin appears to have a permissive role in MPS in the presence of elevated AAs, and plays a clear role in reducing MPB independent of AA availability.

Keywords Amino acids · Insulin · Meta-analysis · Muscle protein breakdown $\cdot$ Muscle protein synthesis $\cdot$ Systematic review
Abbreviations
AA Amino acid
MPB Muscle protein breakdown
MPS Muscle protein synthesis
NB Net balance
WMD Weighted mean difference

\section{Introduction}

In humans, proteins constitute approximately $15 \%$ of body weight [1]. They are the primary macro-nutrient that forms skeletal muscle, which in turn contains approximately 30 $45 \%$ of total body protein and contributes to $20-35 \%$ of whole-body protein turnover. Both amino acids (AAs) and insulin have been shown to play crucial roles in regulating diurnal changes in skeletal muscle protein turnover [2], and 
imbalances between the rates of muscle protein synthesis (MPS) and muscle protein breakdown (MPB) have important consequences for muscle size, quality and function [3]. 'Sarcopenia' describes the loss of skeletal muscle mass and strength that occurs with advancing age [4]. The ageing process itself is characterised by the incipient development of sarcopenia, where a steady decline in lean mass (and associated function) of approximately $1 \%$ per year beyond 60 years of age has been reported [5]. Because of associated frailty, sarcopenia leads to decreased quality of life and health, characterised by poor mobility, sedentarism, an increased risk of falls and poor recovery from illness $[6,7]$.

Data from epidemiological and experimental studies have reported that type 2 diabetes is related to poor muscle strength and function, with an accelerated rate of decline in muscle quality and strength in older individuals of up to $30 \%$ [8]. In view of the rising prevalence of diabetes and the metabolic sequelae of age-related sarcopenia, there has been increased interest in the mechanisms by which type 2 diabetes exacerbates the age-related decline in muscle mass. Furthermore, since skeletal muscle is an important site for glucose disposal, quantitative reductions in appendicular muscle volume might potentially adversely affect glucose disposal and metabolism [9]. Increased understanding of the endocrine factors that regulate muscle mass is therefore important in terms of glycaemic control and countering sarcopenia.

Many patients with type 2 diabetes eventually require insulin to achieve optimal glucose targets, as the ability to produce endogenous insulin from pancreatic beta cells progressively declines [10]. However, insulin therapy is associated with weight gain [11, 12], predominantly fat mass [13], which increases insulin resistance and necessitates the use of higher doses of insulin at the expense of further weight gain. The exact role of insulin in human skeletal muscle metabolism, however, continues to raise debate. Although animal studies have reported that insulin promotes MPS, these studies have mainly been performed with growing animals $[14,15]$. The role of insulin in adult human skeletal muscle is more complex and subject to interplay between other factors such as AA availability, muscle blood flow and microvascular recruitment $[16,17]$. This has led to various studies reporting opposing conclusions when it comes to the relationship between insulin and human skeletal muscle protein turnover [16-22]. We therefore undertook a systematic review and meta-analysis in an attempt to clarify the role of insulin in regulating muscle metabolism in humans.

\section{Methods}

A systematic search of MEDLINE and EMBASE, and of reviews and the references lists of relevant articles, was conducted using the terms 'muscle protein synthesis' OR 'fractional synthesis rate' OR 'muscle protein anabolism' OR 'muscle protein metabolism', paired with the term 'insulin'.

Study selection We searched English-language studies published between 1946 and November 2013. Peerreviewed papers investigating the role of insulin on MPS and/or MPB were selected. All experimental studies that reported changes in muscle protein metabolism in humans in response to interventions with insulin were selected for the systematic review, regardless of the method of evaluation. Both the two-pool (compartment) and three-pool methods were used in reporting muscle protein metabolism, and both provide qualitatively comparable changes in protein metabolism from blood and intracellular enrichment of phenylalanine [21]. In the two-pool (artery and vein) model, phenylalanine enters and leaves the limb via the artery and vein, respectively. The rate of disappearance of phenylalanine from the artery is used to estimate MPS, and is derived from measurements of the rate of MPB and net balance (NB); MPB is determined by the rate of appearance of phenylalanine in the vein (i.e. the dilution of tracer enrichment across the limb), while NB is simply the concentration difference of phenylalanine across the limb. In the threepool (artery, vein and muscle) model, phenylalanine enters and leaves the limb as above. The unidirectional flow of free phenylalanine from the artery to the intramuscular compartment is determined as the rate of its inward transport. The rate of the intracellular appearance of phenylalanine defines the rate of release from MPB. Since phenylalanine is not oxidised by skeletal muscle, the rate of its intracellular use corresponds to the rate of use for MPS [22].

In the studies we reviewed, the two-pool model was the most commonly used analytical method and phenylalanine was the most commonly used AA tracer. Therefore, in order to allow comparable quantitative analysis of the studies eligible for the systematic review and to prevent significant heterogeneity, the meta-analysis only included studies that used the two-pool model to analyse phenylalanine data, as opposed to other analytical methods or other AA tracers (e.g. leucine). Three-pool data were included in the systematic review. Due to a large overlap between studies that reported two- and three-pool data $(n=10)$ and the significantly fewer studies that exclusively reported three-pool data $(n=5)$, we did not perform a meta-analysis of the three-pool data.

Data extraction and statistical analysis All studies meeting the inclusion criteria were reviewed. The primary outcome 
was the change in MPS and/or MPB in response to insulin intervention. Published data were extracted from the studies and SDs of the means were calculated. Because of similar reporting methods of measurement between the included studies, random-effects models were used to calculate weighted mean differences (WMDs), 95\% CIs and corresponding $p$ values. Heterogeneity between studies was assessed using the $I^{2}$ statistic, which describes the percentage of total variation across studies that is a result of heterogeneity rather than chance [23]. Because AAs are the principal substrate for protein synthesis, subgroup analyses were performed based on different levels of AA delivery to the muscle, and hence the amount available (increased, no change or decreased) for protein metabolism. Another subgroup analysis was performed with studies involving populations with diabetes. In these studies, AA delivery did not change. Meta-regression analysis was conducted to test for differences in the pooled estimates between subgroups, and to test whether pooled estimates differed according to other covariants (e.g. insulin concentration levels achieved, age or lean body mass).

Publication bias was assessed by examining a funnel plot as a function of effect size. Statistical tests for the meta-analyses were performed using the statistical package STATA 13.0 (StataCorp, College Station, TX, USA).

\section{Results}

Search results After removal of duplicates, 646 articles were retrieved from the search and the reference lists of selected articles (Fig. 1). Title and abstract screening led to 455 articles being excluded because of irrelevance (i.e. in-vitro studies, studies investigating liver protein metabolism) and a further 87 articles were excluded because the studies were conducted in animal skeletal muscle. A total of 104 potentially relevant articles were identified and evaluated in greater detail. Of these, a further 60 articles were excluded. The main reasons for exclusion were: (1) the studies assessed the role of nutritional interventions but not insulin per se; and (2) the articles were reviews rather than research study articles. A total of 44 articles encompassing 75 studies met the criteria for the systematic review. Of these 44 articles, 13 (containing 25 studies) were included in the meta-analysis, as they represented the largest group that contained quantitatively comparable data. All of the 25 studies used phenylalanine as the AA tracer, reported MPS/MPB in units of nmol (100 ml leg vol. $)^{-1} \mathrm{~min}^{-1}$ and used the two-pool (arteriovenous balance) approach to estimate outcome variables.
Meta-analysis of study characteristics A total of 13 articles [16, 19-21, 24-32], containing 25 experimental studies using different insulin concentrations, were identified for inclusion in the meta-analysis (Table 1); all analysed the effect of insulin on both MPS and MPB (Table 1). A total of 173 individuals were included in these studies. A total of 13 studies involved young adults, and three of these 13 studies involved individuals with diabetes. Eight studies involved healthy older individuals, and there were no age data available for four studies. The average age of the participants ranged from 18 to 74 years. The majority of the studies (20 studies) used local intra-arterial insulin infusion to limit the development of systemic hypoglycaemia and an obligatory infusion of glucose, also to limit hypoaminoacidaemia [33]. This is of crucial importance, especially when supraphysiological insulin concentrations are used. Systemic insulin concentrations varied between 62.5 and $861.2 \mathrm{pmol} / \mathrm{l}$. AA delivery (concentration of AA in the artery $\times$ arterial blood flow) was maintained in 14 studies and increased in eight studies. However, as a direct consequence of systemic insulin administration (see discussion below), circulating AAs were decreased in three studies.

Effect of insulin on MPS The meta-analysis data were pooled from 13 trials (25 studies or comparisons) involving 173 individuals. The WMD for MPS was 3.90 $(95 \%$ CI $-0.74,8.55 ; p=0.71)$. Analysing the studies based on AA delivery revealed increased MPS (WMD 13.44 [95\% CI 4.07, 22.81], $p<0.01$ ) in the studies where AA delivery was increased (eight studies, 63 individuals). However, MPS did not significantly change when AA delivery was reduced (three studies; 22 participants; WMD 1.57 [95\% CI $-3.97,7.12], p=0.58$ ) or maintained at baseline (11 studies; 73 participants; WMD 2.00 [95\% CI -5.28, 9.28], $p=0.59$ ). Studies involving individuals with diabetes (three studies, 15 individuals) showed significant reductions in MPS in response to insulin, even though AA delivery was maintained (WMD -6.67 [95\% CI $-12.69,-0.66], p<0.05$; Fig. 2).

On meta-regression analysis, the estimate size (WMD) was significantly different between subgroups based on AA availability $(p=0.001)$.

The $I^{2}$ for the overall effect of insulin on MPS was $49 \%(p=0.003)$. This significant moderate heterogeneity seemed to be primarily due to heterogeneity within the increased AA subgroup $\left(I^{2} 59 \% ; p=0.018\right)$. The other subgroups showed non-significant $p$ values for heterogeneity, suggesting greater consistency among these studies compared with the overall MPS data (subgroups: 
Fig. 1 Flow chart representing the article-retrieval process and outlining the articles that met the criteria for systematic review and meta-analysis. Only studies using the two-pool model and phenylalanine tracers were included in the meta-analysis

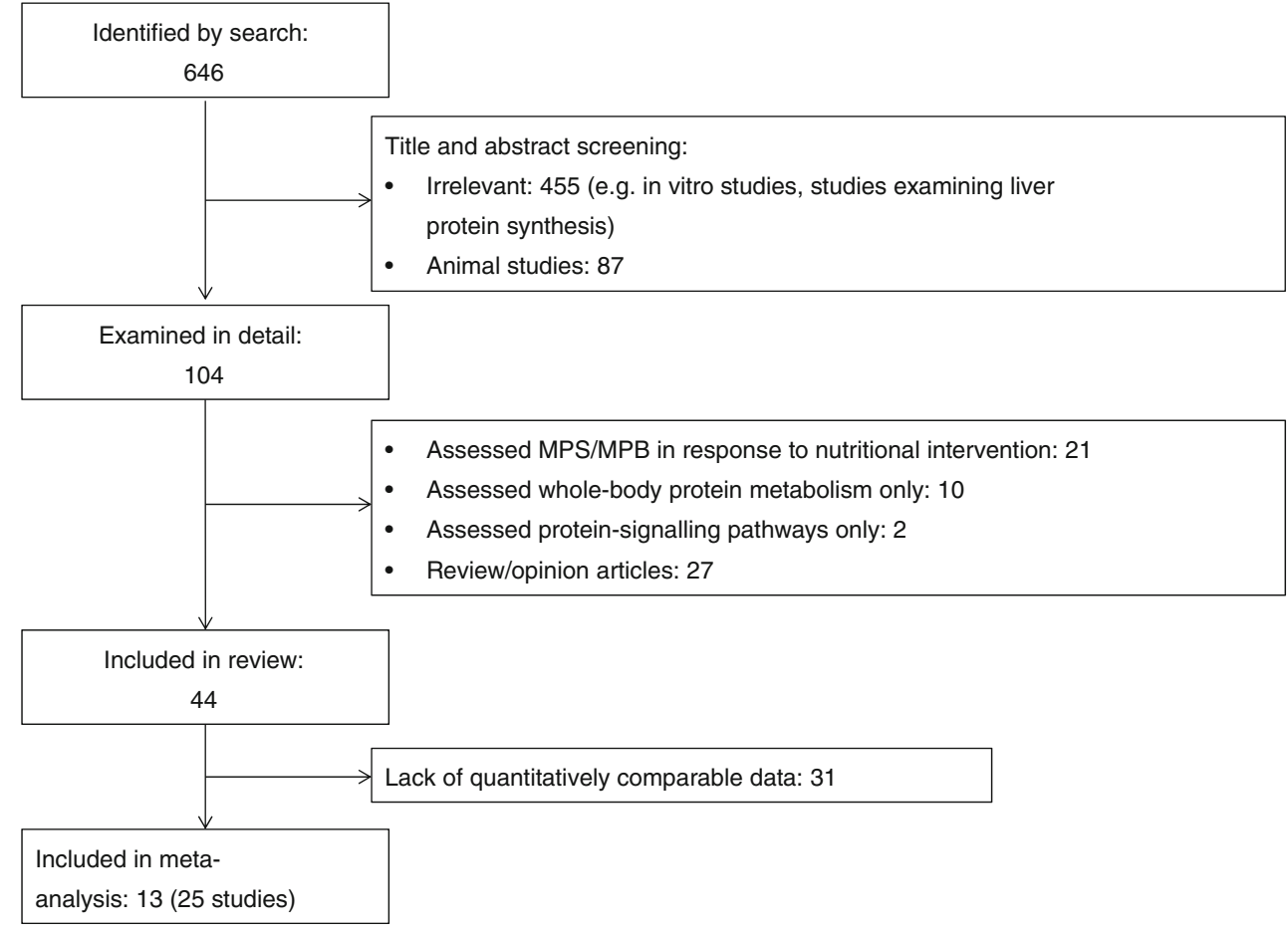

maintained AA delivery: $I^{2} 21 \% ; p=0.241$; decreased AA delivery: $I^{2} 0 \% ; p=0.811$; individuals with diabetes: $\left.I^{2} 0 \% ; p=0.605\right)$.

Effect of insulin on MPB Data were pooled from the same 25 studies as for MPS. The WMD for MPB was -15.46 (95\% CI -19.74, -11.18; $p<0.0001$; Fig. 3). AA availability did not have a significant impact on the estimated size of MPB $(p=0.754)$. The $I^{2}$ for overall insulin effect on MPB was $13 \%(p=0.282)$, pointing to non-significant heterogeneity (Fig. 3).

Effect of insulin on protein NB In a further pooled analysis of all 25 studies, insulin was found to significantly increase NB protein acquirement (WMD 20.09 [95\% CI 15.93, 24.26], $p<0.0001$; Fig. 4).

Meta-regression analysis of other variables A metaregression analysis was conducted to test whether other confounding variables of interest would have any effect on the WMD (e.g. concentration of insulin infusion, age and lean body mass, where available). Differences in the infused insulin concentrations had no effect on MPS $(p=0.955)$, MPB $(p=0.713)$ or NB $(p=0.621)$. There was also no effect for age differences $(p=0.480, p=$ 0.159 and $p=0.610$, respectively) or for changes in lean body mass $(p=0.433, p=0.936$ and $p=0.617$, respectively).
Publication bias Funnel plots of the insulin effect on both MPS and MPB against SE showed no publication bias (see electronic supplementary material [ESM] Figs 1, 2).

Other data From the articles reviewed, 15 studies used three-pool data [21, 25, 28, 30-35]. Two-pool data from ten of these studies were included in the meta-analysis, which by and large reported similar results with quantitatively different displays. All five studies that exclusively reported three-pool data showed that insulin improved NB protein acquirement [25, 33-35]. The increment in NB in these studies was primarily driven by either a reduction in MPB [34] or an increase in MPS [25, 33-35]. None of these studies involved a reduction in AA availability. Two of the studies reported an increase in MPS in an experimentally induced hyperinsulinaemic state in seemingly insulin-resistant individuals with severe burns who were being treated in a high-dependency unit setting [33, 35]. A summary of the systematic review of three-pool data is presented in Table 2.

\section{Discussion}

Systemic insulin infusion leads to hypoglycaemia and reduced AA availability (hypoaminoacidaemia) for protein synthesis. To overcome these consequences, glucose and AA are coinfused to maintain target glycaemia and AA availability. 


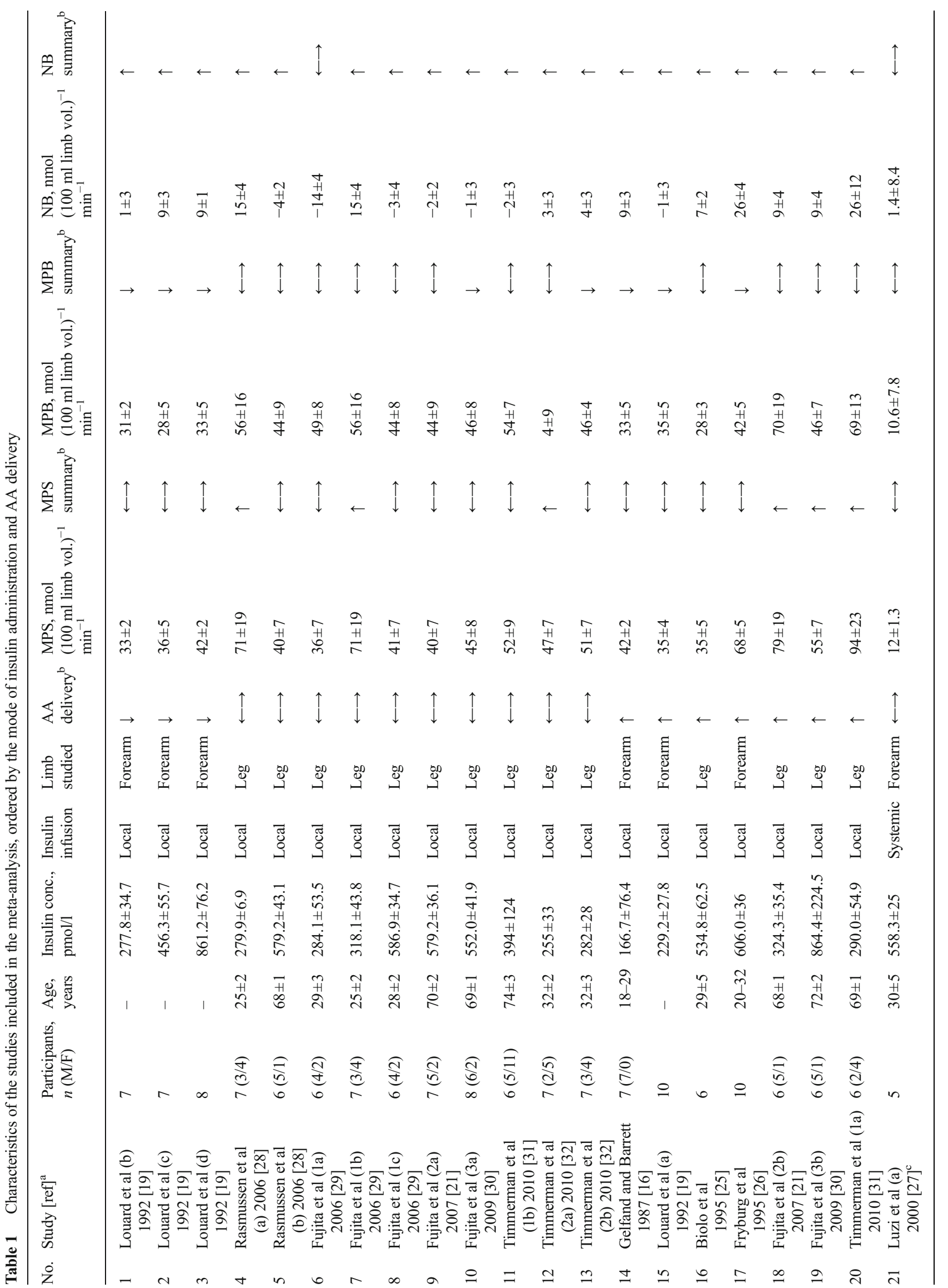




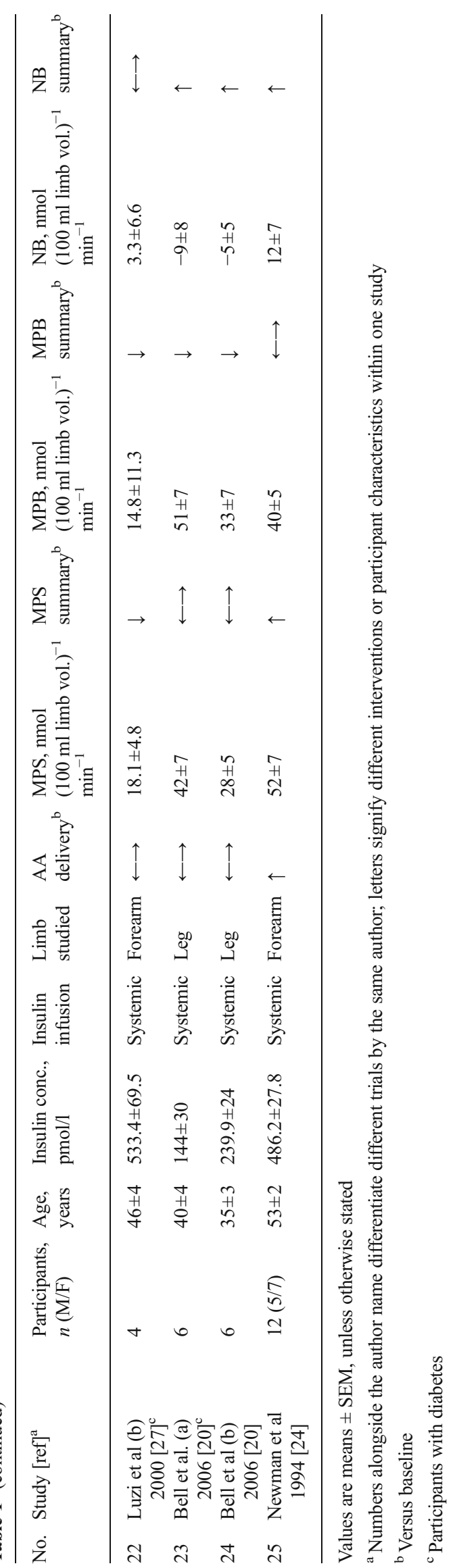


Fig. 2 Forest plot of randomeffects meta-analysis of WMD (95\% CI) on the effect of insulin on MPS. No significant increase in MPS was seen overall $(p=$ 0.710 ). When stratified for AA delivery, increased MPS was seen where AA was increased $(p<0.01)$, but no difference was observed where AA delivery was maintained $(p=0.59)$ or decreased $(p=0.58)$. In individuals with insulin resistance or diabetes (IR), MPS was significantly reduced $(p<0.05)$ despite maintained AA delivery. Numbers alongside the author name differentiate different trials by the same author; letters signify different interventions or participant characteristics within one study

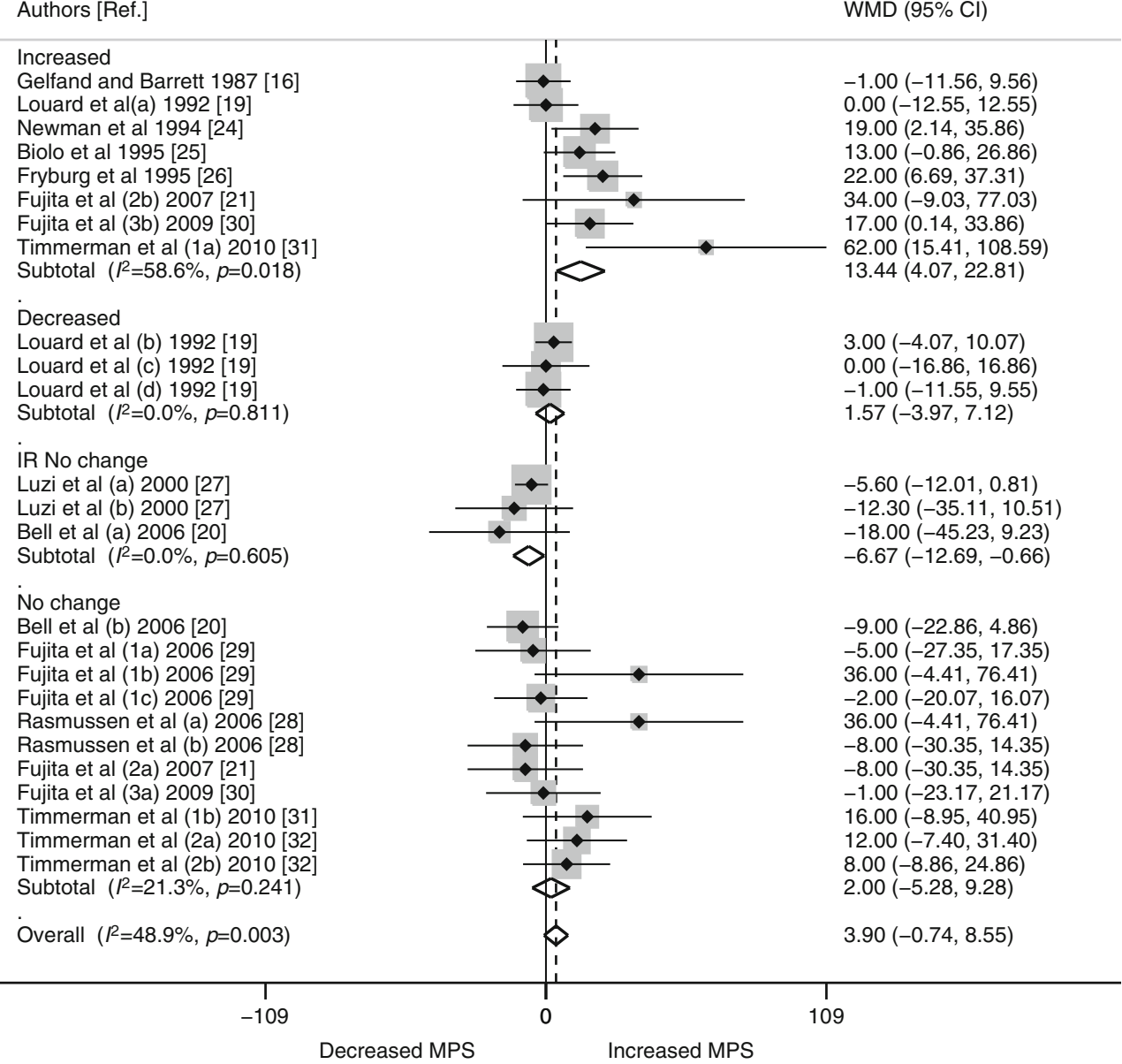

Local intra-arterial insulin infusion appears to limit the effect of systemic hypoglycaemia and hypoaminoacidaemia, thereby avoiding the need for obligatory glucose (or AA) coinfusion [33].

Meta-analysis of these 25 studies showed no significant effect of insulin on MPS. Subgroup analysis, however, revealed that in healthy individuals, the effect of insulin on MPS only becomes significant when AA delivery to the skeletal muscle is increased. These results have been replicated by other researchers in studies where coinfusion of AA and insulin has successfully increased AA delivery to muscle $[30,31,36-42]$. In a study by Fujita et al, exercising for 45 min successfully increased AA delivery and MPS compared with non-exercising controls, although exercise per se has acute anabolic effects on muscle MPS [21]. In some studies, however, increased AA delivery has not produced an insulininduced increase in MPS [16, 43]. This is probably because the rises in AA delivery were very minimal and achieved mainly through increased blood flow, rather than an increased AA concentration. Older people display resistance to the anabolic effect of insulin compared with their younger counterparts, possibly through mechanisms related to endothelial dysfunction, reduced tissue perfusion and blunted anabolic signalling, rather than reduced glucose tolerance [30]. However, in the presence of increased AA delivery, insulin seems to preserve its anabolic effect in healthy older people. This seems to be the case whether the increments in AA delivery to muscle are achieved via physiological [24, 38] or supraphysiological [30] concentrations of insulin, pharmacologically using sodium nitroprusside [31] or via exercise [21].

Increased insulin concentrations within the postprandial range do not appear to affect MPS. A previous study reported that, with incremental increases in AA concentrations, MPS responded positively to insulin concentrations of $139.0-194.5 \mathrm{pmol} / \mathrm{l}$, rising by $22 \%$ from baseline and by $72 \%$ when higher AA concentrations were given [37]. On the other hand, another study reported that, in the presence of fixed concentrations of AA, increasing insulin concentration from $34.7 \mathrm{pmol} / 1$ to $500.0 \mathrm{pmol} / \mathrm{l}$ did not produce any further significant increments in MPS [39]. Insulin had no effect on MPS when AA delivery was unchanged from baseline. These results are also supported by other studies [17, 20, 
Fig. 3 Forest plot of randomeffects meta-analysis of WMD $(95 \% \mathrm{CI})$ on the effect of insulin on MPB. A significant reduction in MPB was seen overall $(p<0.0001)$. Numbers alongside the author name differentiate different trials by the same author; letters signify different interventions or participant characteristics within one study
Authors [Ref.]

WMD $(95 \% \mathrm{Cl})$

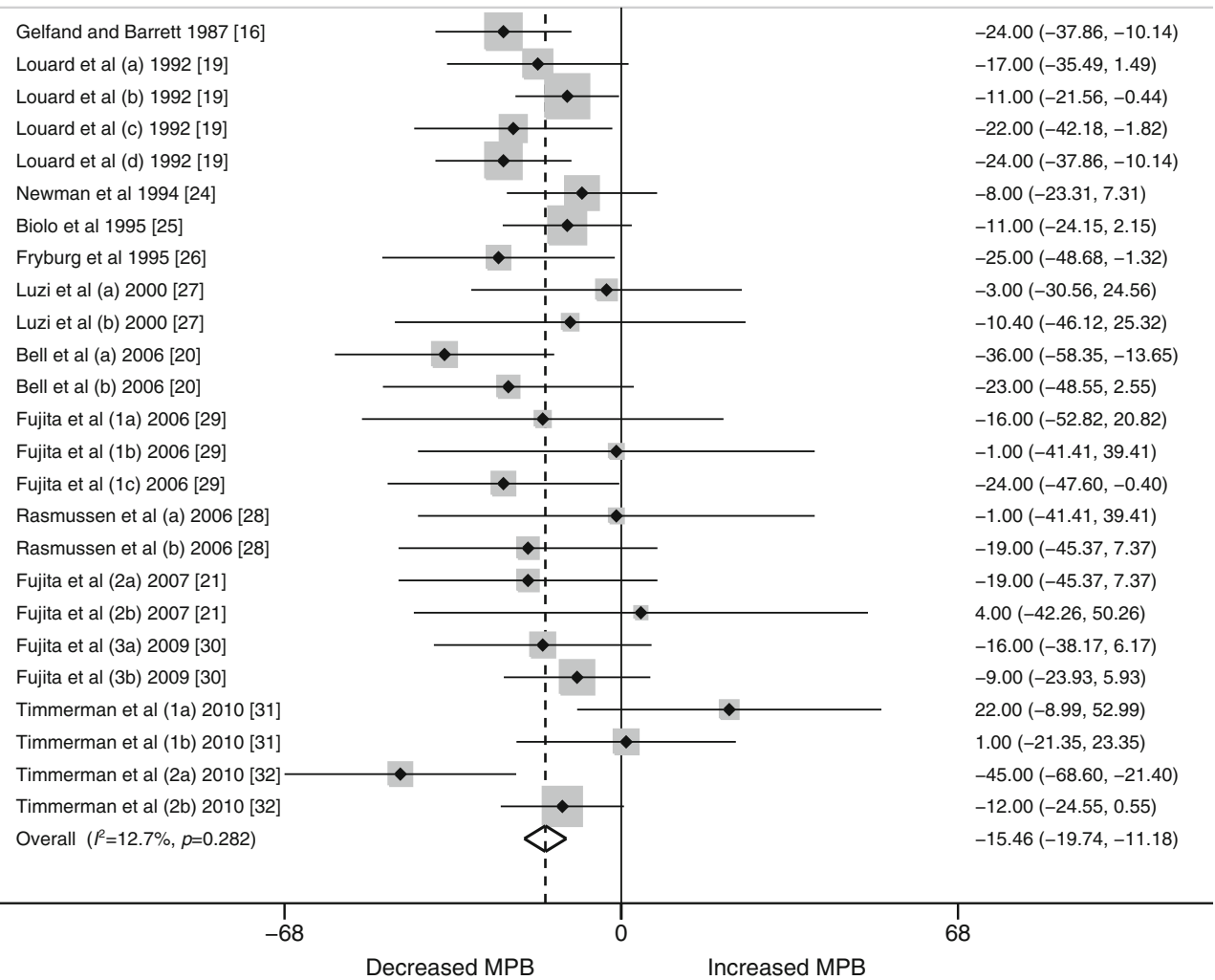

28-31, 34, 44]. In all healthy human studies where AA availability has been reduced, MPS has been reduced or remained unchanged $[18,19,37,45,46]$, even in the presence of supraphysiological concentrations of insulin [18].

The meta-analysis of the 25 studies showed that insulin exerts its regulation of lean muscle mass principally via an anticatabolic effect in reducing MPB. This is more evident when looking at the data on NB, which showed a positive effect on muscle mass. Therefore, the proanabolic capabilities of insulin are predominantly driven by its ability to attenuate skeletal MPB, rather than any positive effect on MPS. This finding is in agreement with assessments from other researchers $[16,29]$.

The insulin-induced reduction in MPB appears to be more potent when AAs are scarce. These findings are consistent with other studies that have reported a reduction in response to insulin [30, 32, 37, 39, 42, 44] with the exception of three studies, which did not observe any significant change in MPB [45, 47, 48]. This might well be due to anabolic resistance to insulin in a relatively older study population and the presence of diabetes mellitus. Interestingly, this maximal inhibition of
MPB by insulin occurs in response to very modest elevations in insulin concentration (i.e. to $104.2 \mathrm{pmol} / \mathrm{l}$ ) [44].

Diabetes has been reported to attenuate the positive effect of insulin on MPS in the presence of maintained AA delivery [17, 20, 49]. However, in response to long-term intensive s.c. insulin treatment, Halvatsiotis et al found no difference in mitochondrial, sarcoplasmic or mixed MPS compared with healthy controls who did not receive insulin [50]. Whether increased AA intake in insulin-treated patients with diabetes will lead to increased muscle mass is not known. However, given the facilitative role of insulin in maintaining muscle mass, particularly in the presence of increased AA availability, it is tempting to speculate on the need to advise patients with diabetes who are on insulin treatment to increase their AA intake in order to harness the positive effects of insulin on muscle metabolism. In critically ill patients, where significant insulin resistance is expected, increases in MPS have been observed, but only when supraphysiological concentrations of insulin have been used [33, 51]. In a study of individuals with obesity [40] and heart failure [42], MPB has been shown to reduce in response to insulin. 
Fig. 4 Forest plot of randomeffects meta-analysis of WMD $(95 \% \mathrm{CI})$ on the effect of insulin on protein NB kinetics. Insulin had a strong positive effect on NB protein acquirement overall $(p<0.0001)$. Studies are ordered chronologically. Numbers alongside the author name differentiate different trials by the same author; letters signify different interventions or one study participant characteristics within

WMD $(95 \% \mathrm{Cl})$

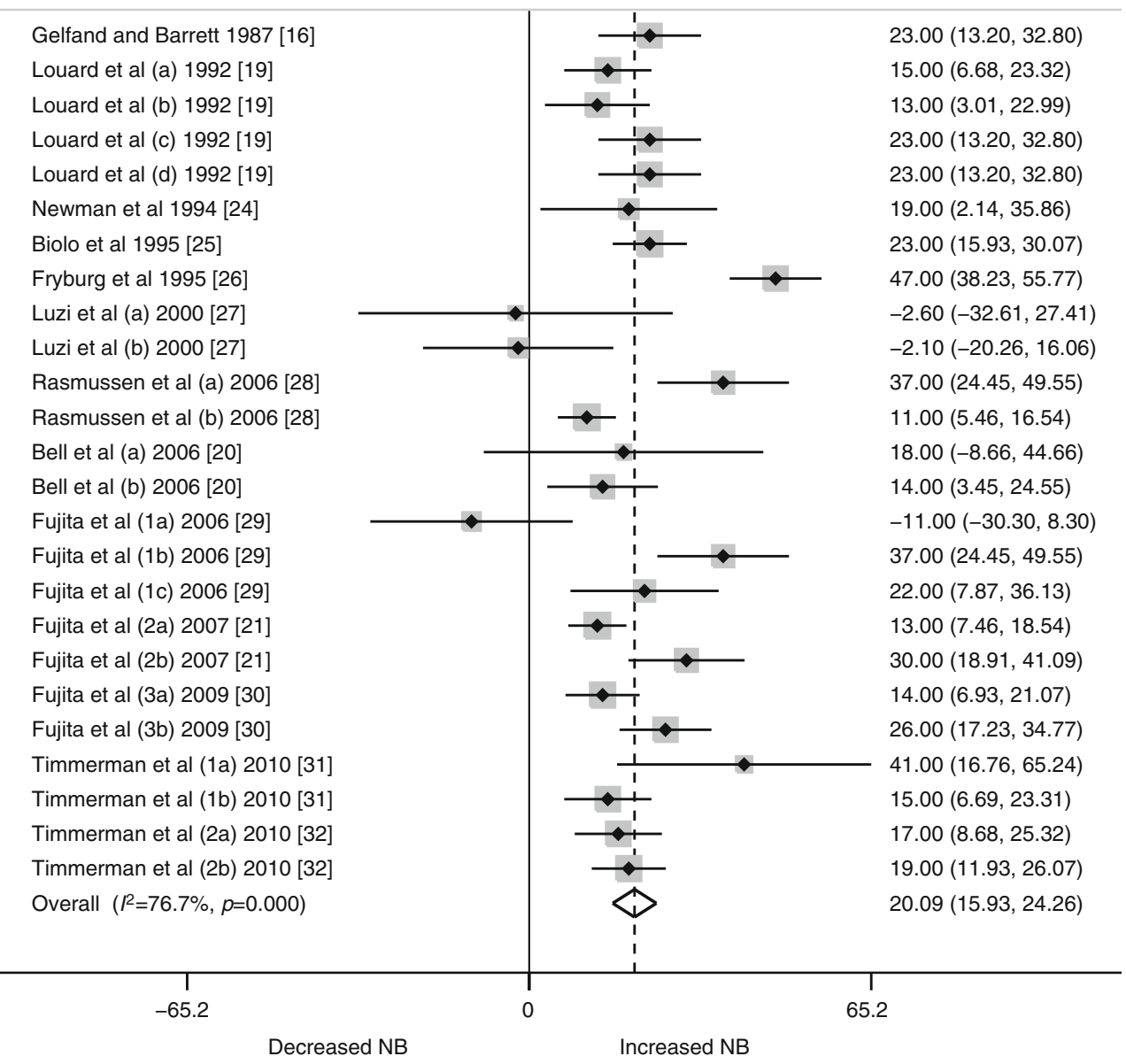

This reduction, however, was significantly less than that observed in healthy controls. Clearly, further studies are required to fully understand the role of insulin resistance in regulating MPS and MPB in type 2 diabetes.

This review has several limitations. Different methods of estimating skeletal muscle protein metabolism and the lack of available primary data made it difficult to perform a full quantitative assessment by meta-analysis for all studies that met the inclusion criteria of the systematic review. We also acknowledge that the use of multiple studies per publication means that pooling of the studies is not entirely independent. In addition, since this is a meta-analysis of experimental studies, a full assessment of bias - as would normally be conducted in meta-analyses of randomised controlled trials [52] (e.g. sequence generation and allocation concealment to check for selection bias; blinding to check for possible performance bias, incomplete outcome data, selective reporting bias and other sources of bias) - could not be performed, as none of the studies used these methods for patient allocation. Furthermore, since all of the studies were of similar size, the funnel plot analysis might not be completely reliable in informing us of any publication bias.

In summary, this systematic review and meta-analysis suggests that the main role of insulin in human skeletal muscle anabolism is facilitative and is influenced by the rate of AA delivery. In situations where AA delivery is unchanged, supraphysiological concentrations of insulin are needed to achieve skeletal muscle anabolism. However, the role of insulin in reducing MPB is clearly evident in most studies. This effect is blunted in older people and in those with insulin resistance. This resistance is likely to be related to impaired insulin signalling of muscle protein metabolism and endothelial dysfunction, rather than to glucose intolerance. Further evidence is required to translate these findings into strategies to maximise muscle mass among patients with insulin-treated diabetes. 


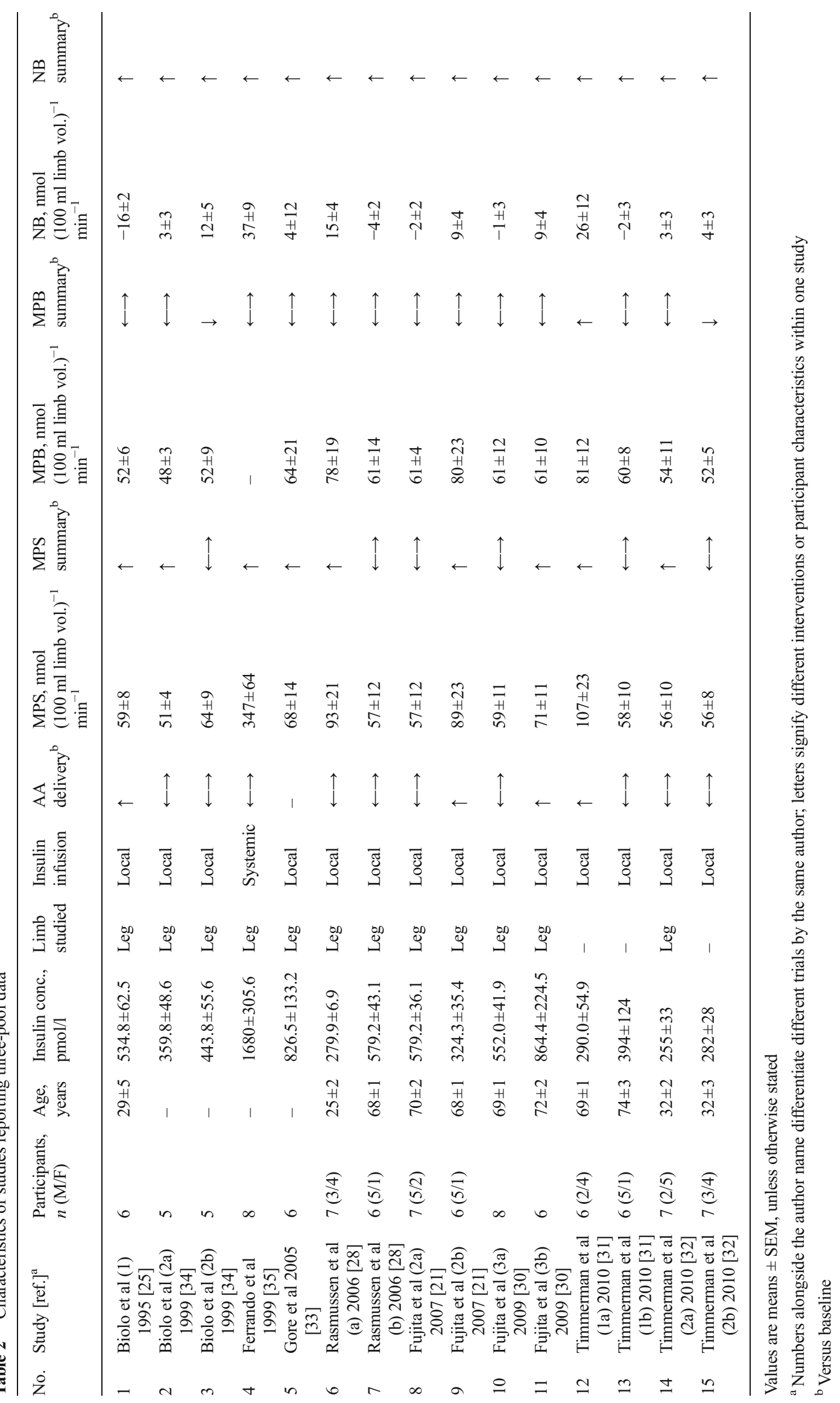


Acknowledgements Some of the data in this manuscript were presented as a poster abstract at the Diabetes UK Annual Meeting, London, March 2015 .

Funding HA received research fellowship funding from the Research, Development and Innovation department at the Derby Teaching Hospital and the University of Nottingham. The authors declare no other sources of funding related to this manuscript.

Duality of interest The authors declare that there is no duality of interest associated with this manuscript.

Contribution statement HA and II constructed the concept of the study. HA systematically reviewed the literature and acquired the data. HA, II, PJA and KS analysed the data. HA wrote the first draft of the manuscript. All authors contributed to interpretation of the data, revised the article critically for important intellectual content and approved the final version of the paper to be published. II is responsible for the integrity of the work as a whole and is the guarantor of this work.

\section{References}

1. Bier DM (1989) Intrinsically difficult problems: the kinetics of body proteins and amino acids in man. Diabetes Metab Rev 5:111-132

2. Prod'homme M, Rieu I, Balage M, Dardevet D, Grizard J (2004) Insulin and amino acids both strongly participate to the regulation of protein metabolism. Curr Opin Clin Nutr Metab Care 7:71-77

3. McNurlan MA, Garlick PJ (1989) Influence of nutrient intake on protein turnover. Diabetes Metab Rev 5:165-189

4. Cruz-Jentoft AJ, Landi F, Schneider SM et al (2014) Prevalence and intervention for sarcopenia in ageing adults: a systematic review. Report of the International Sarcopenia Initiative (EWGSOP and IWGS). Age Ageing 43:748-759

5. Atherton PJ, Etheridge T, Watt PW et al (2010) Muscle full effect after oral protein: time-dependent concordance and discordance between human muscle protein synthesis and mTORC1 signaling. Am J Clin Nutr 92:1080-1088

6. Wolfson L, Judge J, Whipple R, King M (1995) Strength is a major factor in balance, gait, and the occurrence of falls. J Gerontol A Biol Sci Med Sci 50:64-67

7. Tinetti ME, Williams CS (1997) Falls, injuries due to falls, and the risk of admission to a nursing home. N Engl J Med 337:1279-1284

8. Park SW, Goodpaster BE, Lee JS et al (2009) Excessive loss of skeletal muscle mass in older adults with type 2 diabetes. Diabetes Care 32:1993-1997

9. Mavros Y, Kay S, Anderberg KA et al (2013) Changes in insulin resistance and $\mathrm{HbA}_{1 \mathrm{c}}$ are related to exercise-mediated changes in body composition in older adults with type 2 diabetes: interim outcomes from the GREAT2DO trial. Diabetes Care 36:2372-2379

10. Prospective UK (1995) Diabetes Study Group. UK Prospective Diabetes Study 16. Overview of 6 years' therapy of type II diabetes: a progressive disease. Diabetes 44:1249-1258

11. Koro CE, Bowlin SJ, Bourgeois N, Fedder DO (2004) Glycemic control from 1988 to 2000 among U.S. adults diagnosed with type 2 diabetes: a preliminary report. Diabetes Care 27:17-20

12. Biesenbach G, Raml A, Alsaraji N (2006) Weight gain and insulin requirement in type 2 diabetic patients during the first year after initiating insulin therapy dependent on baseline BMI. Diabetes Obes Metab 8:669-673

13. Packianathan IC, Fuller NJ, Peterson DB, Wright A, Coward WA, Finer N (2005) Use of a reference four-component model to define the effects of insulin treatment on body composition in type 2 diabetes: the 'Darwin study'. Diabetologia 48:222-229

14. Pain VM, Albertse EC, Garlick PJ (1983) Protein metabolism in skeletal muscle, diaphragm, and heart of diabetic rats. Am J Physiol 245:E604-E610

15. Garlick PJ, Grant I (1988) Amino acid infusion increases the sensitivity of muscle protein synthesis in vivo to insulin. Effect of branched-chain amino acids. Biochem J 254:579-584

16. Gelfand RA, Barrett EJ (1987) Effect of physiologic hyperinsulinemia on skeletal muscle protein synthesis and breakdown in man. J Clin Invest 80:1-6

17. Pacy PJ, Nair KS, Ford C, Halliday D (1989) Failure of insulin infusion to stimulate fractional muscle protein synthesis in type I diabetic patients; anabolic effect of insulin and decreased proteolysis 38:612-624

18. Denne SC, Liechty EA, Liu YM, Brechtel G, Baron AD (1991) Proteolysis in skeletal muscle and whole body in response to euglycemic hyperinsulinemia in normal adults. Am J Physiol 261:E809-E814

19. Louard RJ, Fryburg DA, Gelfand RA, Barrett EJ (1992) Insulin sensitivity of protein and glucose metabolism in human forearm skeletal muscle. J Clin Invest 90:2348-2354

20. Bell JA, Volpi E, Fujita S et al (2006) Skeletal muscle protein anabolic response to increased energy and insulin is preserved in poorly controlled type 2 diabetes. J Nutr 136:1249-1255

21. Fujita S, Rasmussen BB, Cadenas JG et al (2007) Aerobic exercise overcomes the age-related insulin resistance of muscle protein metabolism by improving endothelial function and Akt/mammalian target of rapamycin signaling. Diabetes 56:1615-1622

22. Biolo G, Fleming RY, Maggi SP, Wolfe RR (1995) Transmembrane transport and intracellular kinetics of amino acids in human skeletal muscle. Am J Physiol 268:E75-E84

23. Higgins JP, Thompson SG, Deeks JJ, Altman DG (2003) Measuring inconsistency in meta-analyses. BMJ 327:557-560

24. Newman E, Heslin MJ, Wolf RF, Pisters PW, Brennan MF (1994) The effect of systemic hyperinsulinemia with concomitant amino acid infusion on skeletal muscle protein turnover in the human forearm. Metabolism 43:70-78

25. Biolo G, Declan Fleming RY, Wolfe RR (1995) Physiologic hyperinsulinemia stimulates protein synthesis and enhances transport of selected amino acids in human skeletal muscle. J Clin Invest 95:811-819

26. Fryburg DA, Jahn LA, Hill SA, Oliveras DM, Barrett EJ (1995) Insulin and insulin-like growth factor-I enhance human skeletal muscle protein anabolism during hyperaminoacidemia by different mechanisms. J Clin Invest 96:1722-1729

27. Luzi L, Piceni Sereni L, Spessot M et al (2000) Postabsorptive muscle protein metabolism in type 1 diabetic patients after pancreas transplantation. Acta Diabetol 37:219-224

28. Rasmussen BB, Fujita S, Wolfe RR et al (2006) Insulin resistance of muscle protein metabolism in aging. FASEB J 20:768-769

29. Fujita S, Rasmussen BB, Cadenas JG, Grady JJ, Volpi E (2006) Effect of insulin on human skeletal muscle protein synthesis is modulated by insulin-induced changes in muscle blood flow and amino acid availability. Am J Physiol Endocrinol Metab 291:E745-E754

30. Fujita S, Glynn EL, Timmerman KL, Rasmussen BB, Volpi E (2009) Supraphysiological hyperinsulinaemia is necessary to stimulate skeletal muscle protein anabolism in older adults: evidence of a true age-related insulin resistance of muscle protein metabolism. Diabetologia 52:1889-1898

31. Timmerman KL, Lee JL, Fujita S et al (2010) Pharmacological vasodilation improves insulin-stimulated muscle protein anabolism but not glucose utilization in older adults. Diabetes 59:2764-2771

32. Timmerman KL, Lee JL, Dreyer HC et al (2010) Insulin stimulates human skeletal muscle protein synthesis via an indirect mechanism 
involving endothelial-dependent vasodilation and mammalian target of rapamycin complex 1 signaling. J Clin Endocrinol Metab 95:3848-3857

33. Gore DC, Herndon DN, Wolfe RR (2005) Comparison of peripheral metabolic effects of insulin and metformin following severe burn injury. J Trauma Inj Infect Crit Care 59:316-323

34. Biolo G, Williams BD, Fleming RY, Wolfe RR (1999) Insulin action on muscle protein kinetics and amino acid transport during recovery after resistance exercise. Diabetes 48:949-957

35. Ferrando AA, Chinkes DL, Wolf SE, Matin S, Herndon DN, Wolfe RR (1999) A submaximal dose of insulin promotes net skeletal muscle protein synthesis in patients with severe burns. Ann Surg 229:11-18

36. Hillier TA, Fryburg DA, Jahn LA, Barrett EJ, Teresa A (1998) Extreme hyperinsulinemia unmasks insulin's effect to stimulate protein synthesis in the human forearm. Am J Physiol Endocrinol Metab 247:E1067-E1074

37. Nygren J, Nair KS (2003) Differential regulation of protein dynamics in splanchnic and skeletal muscle beds by insulin and amino acids in healthy human subjects. Diabetes 52:1377-1385

38. Guillet C, Prod'homme M, Balage M et al (2004) Impaired anabolic response of muscle protein synthesis is associated with S6K1 dysregulation in elderly humans. FASEB J 18:1586-1587

39. Greenhaff PL, Karagounis LG, Peirce N et al (2008) Disassociation between the effects of amino acids and insulin on signaling, ubiquitin ligases, and protein turnover in human muscle. Am J Physiol Endocrinol Metab 295:595-604

40. Guillet C, Delcourt I, Rance M et al (2009) Changes in basal and insulin and amino acid response of whole body and skeletal muscle proteins in obese men. J Clin Endocrinol Metab 94:3044-3050

41. Smith GI, Atherton P, Reed DN et al (2009) No major sex differences in muscle protein synthesis rates in the postabsorptive state and during hyperinsulinemia-hyperaminoacidemia in middle-aged adults. J Appl Physiol 107:1308-1315

42. Toth MJ, LeWinter MM, Ades PA, Matthews DE (2010) Impaired muscle protein anabolic response to insulin and amino acids in heart failure patients: relationship with markers of immune activation. Clin Sci (Lond) 119:467-476

43. Heslin MJ, Newman E, Wolf RF, Pisters PW, Brennan MF (1992) Effect of hyperinsulinemia on whole body and skeletal muscle leucine carbon kinetics in humans. Am J Physiol 262:E911-E918

44. Wilkes EA, Selby AL, Atherton PJ et al (2009) Blunting of insulin inhibition of proteolysis in legs of older subjects may contribute to age-related sarcopenia. Am J Clin Nutr 90:1343-1350

45. Arfvidsson B, Zachrisson H, Moller-Loswick AC, Hyltander A, Sandstrom R, Lundholm K (1991) Effect of systemic hyperinsulinemia on amino acid flux across human legs in postabsorptive state. Am J Physiol 260:E46-E52

46. Barazzoni R, Short KR, Asmann Y, Coenen-Schimke JM, Robinson MM, Nair KS (2012) Insulin fails to enhance mTOR phosphorylation, mitochondrial protein synthesis, and ATP production in human skeletal muscle without amino acid replacement. Am J Physiol Endocrinol Metab 303:E1117-E1125

47. Wolf RF, Heslin MJ, Newman E, Pearlstone DB, Gonenne A, Brennan MF (1992) Growth hormone and insulin combine to improve whole-body and skeletal muscle protein kinetics. Surgery 112:284-291, discussion 291-292

48. Bell JA, Fujita S, Volpi E, Cadenas JG, Rasmussen BB (2005) Short-term insulin and nutritional energy provision do not stimulate muscle protein synthesis if blood amino acid availability decreases. Am J Physiol Endocrinol Metab 289:E999-E1006

49. Charlton MR, Balagopal P, Nair KS (1997) Skeletal muscle myosin heavy chain synthesis in type 1 diabetes. Diabetes 46:1336-1340

50. Halvatsiotis P, Short KR, Bigelow M, Nair KS (2002) Synthesis rate of muscle proteins, muscle functions, and amino acid kinetics in type 2 diabetes. Diabetes 51:2395-2404

51. Sakurai Y, Aarsland A, Herndon DN et al (1995) Stimulation of muscle protein synthesis by long-term insulin infusion in severely burned patients. Ann Surg 222:283-297

52. Wang C, Mamza J, Idris I (2015) Biphasic vs basal bolus insulin regimen in type 2 diabetes: a systematic review and meta-analysis of randomized controlled trials. Diabet Med 32:585-594 